\title{
Development of Dynamic Interpersonal Therapy in Complex Care (DITCC): A Pilot Study
}

Amra Rao, Alessandra Lemma, Peter Fonagy, Marta Sosnowska, Matthew Constantinou, Malgorzata Fijak-Koch \& Gilla Gelberg

\begin{abstract}
The paper describes the development of Dynamic Interpersonal Therapy for use with complex depression (DITCC), and a pilot study testing DITCC's effectiveness. The pilot found large pre-post improvements in wellbeing and distress; moderate rates of reliable improvement and clinically significant change; and curvilinear declines in depression and anxiety. Treatment completers and near-completers $(N=19)$ showed a significant curvilinear decline in symptoms of depression (PHQ-9) and anxiety (GAD-7). The results provide preliminary evidence that DITCC can be used as an effective treatment approach for complex depression. However, further research is needed to test its effectiveness in different settings with a larger sample size, using appropriate comparison groups under controlled conditions to further elaborate the short-term and long-term effects.
\end{abstract}

\section{Depression - A cause for concern for the health service}

Depression is the leading cause of disability worldwide and is a major contributor to the overall global burden of disease (Vos et al., 2015). Alongside the early onset (Eaton et al., 2008), it tends to follow chronic or relapsing courses with considerable morbidity, risk and interference with social and occupational functioning (Ustün, Ayuso-Mateos, Chatterji, Mathers, \& Murray, 2004). Literature reviews investigating global variation in the prevalence of major depressive disorders have reported a global prevalence of $4.7 \%$, a lifetime prevalence between 10 and 15\% and a global incidence of 3.0\% (Ferrari et al., 2013; Lépine \& Briley, 2011).

Reoccurrence is common in major depression. In non-clinical cohorts a third of those with at least one episode will have another (Eaton et al., 2008). Such a pattern is observed for over three-quarters of patients in clinical samples (Mueller et al., 1999). The mean number of episodes is reported to be approximately four, with a mean duration of approximately 1417 weeks per episode if mild in severity and 23 weeks if severe (Kessler et al., 2003). Childhood maltreatment, post-treatment residual symptoms, and a history of recurrence have emerged as strong prognostic indicators of risk of relapse and reoccurrence in depression (Buckman et al., 2018).

Co-morbid psychiatric diagnosis is often the case (Kessler et al., 2003). 50\%-90\% of patients with Axis I conditions meet criteria for other DSM IV Axis I or Axis II conditions (Westen, 
Novotny, \& Thompson-Brenner, 2004). Partial treatment response and premature disengagement pose particular challenges to effectiveness of treatment. Stimpson, Agrawal, \& Lewis (2002) estimated that a minimum of $30 \%$ depressed individuals with sub-optimal therapeutic responses experience recurrent treatment failures.

\section{Effectiveness and efficiencies}

Chronic and complex depression is common in primary and secondary care and often present challenges around engagement and recovery (Buszewicz, Griffin, McMahon, Beecham, \& King, 2010; Paykel et al., 2005). Literature on the effectiveness of treatment for depression is emerging (McPherson et al., 2005; Stimpson, Agrawal, \& Lewis, 2002). Many factors such as psychosocial functioning, general health and employment stability impact help seeking behaviour, resilience and recovery. These, alongside caregivers' behaviour add further complexity to the debate on treatments effectiveness (Andrews, 2008, McPherson \& Armstrong, 2009). Many patients with complex depression are likely to require multi-layered, long-term care packages (Abbass, Hancock, Henderson, \& Kisely, 2006; Bower, Gilbody, Richards, Fletcher, \& Sutton, 2006; Hollon \& Ponniah, 2010; Morriss et al., 2010; Paykel et al., 2005; Taylor et al., 2012). Given this complexity, many clinicians and services are working on increasing treatment effectiveness.

Whilst the need for long-term approaches is being increasingly recognised, the current financial pressures on the National Health Service (NHS) in the UK have challenged services to demonstrate both outcomes and efficiencies. Commissioners are increasingly seeking briefer models for psychological therapies whilst patients are asking for more choice between therapies. This poses a particular challenge for the treatment of depression, given its evident complexity. The Tavistock Adult Depression Study (TADS) (Taylor et al., 2012) has demonstrated the effectiveness of a psychoanalytic approach and the importance of follow-up data to consider the sustainability of change. However, the demand and capacity imbalance are increasingly putting pressures on services to cut down the length of the individual treatment and consider more group interventions. Whilst, commissioning of therapies is increasingly informed by National Institute of Clinical Excellence (NICE) guidelines, there is an emerging debate on the research methodologies privileged by NICE (McPherson, Rost, Town, \& Abbass, 2018; Mollon, 2009). In primary care, proportion of investment in CBT recommended by NICE due to its substantial evidence base remains much higher than other therapies such as Interpersonal Psychotherapy (IPT), Counselling for Depression, and Dynamic Interpersonal Therapy (DIT) (Perfect, Jackson, Pybis, \& Hill, 2016; NHS England/Health Education England, 2015 Adult IAPT Workforce Census Report, 2016).

Research evidence shows that, as with all available treatments, many patients do not benefit sufficiently. For example, Roth \& Fonagy (2005) indicate that amongst $50 \%$ who respond 
adequately across treatments, half of them lose gains over the following year. Many clinical services offer and value a variety of therapeutic approaches (Moorey, 2010). Whilst CBT works for many, it is not likely to be an effective treatment of choice for all depressed patients, hence the need for on-going research on treatment options (Barkham, \& t, 2018; Johnsen, \& Friborg, 2015; Pybis, Saxon, Hill, \& Barkam, 2017; Psychological Therapies: Annual Report on the use of IAPT services, England, NHS Digital, 2017-18).

\section{The development of DIT}

Dynamic Interpersonal Therapy (DIT) model for mood disorders (Lemma, Target, \& Fonagy, 2011a), rests on the assumption that the patient's interpersonal context is central to understanding of their behaviour and experience (Lemma et al., 2011a). Like other dynamic models, DIT proposes that entrenched relational patterns prevent new input from the external world that could challenge or update internalised developmental models that otherwise maintain the internal world status quo. DIT facilitates change by enhancing the patient's awareness to such maladaptive entrenched relational patterns.

The two key aims are; (a) to help the patient to understand the connection between their presenting symptoms and what is happening in their relationships by identifying a core, unconscious, repetitive Interpersonal Affective Focus - IPAF (b) to encourage the patients' capacity to reflect on their states of mind to enhance their ability to manage interpersonal difficulties (Lemma et al., 2011a).

DIT addresses character problems in a very limited way and does not go beyond those linked to the maintenance of depression. The model consists of three phases: Phase 1, "the engagement/assessment phase" (sessions 1-4); Phase 2, "the middle phase" (sessions 512); and Phase 3, "the ending phase" (sessions 13-16), each one with its own distinctive strategies (Lemma et al., 2011a).

In its initial pilot study, DIT was shown to bring a significant reduction in reported symptoms. $70 \%$ of patients came below the clinical levels (Lemma, Target, \& Fonagy, 2011b). Evidence is emerging on DIT's effectiveness across a range of settings (Chen \& Dognin, 2017; Chen, Ingenito, Kehn, Nehrig, \& Abraham, 2017; Delfstra \& Rooij, 2015; Dognin \& Chen, 2018; Douglas, Ablett-Tate, \& Chadd, 2016; Leonidaki, Lemma \& Hobbis, 2016; Lemma \& Fonagy, 2013). A randomized controlled trial had been completed, awaiting results.

\section{Application of DIT in Complex Care - DITCC}

Whilst evidence for effectiveness of DIT is emerging, the need to adapt the model for complex depression with features of chronicity, co-morbidity and severity arose from clinical observations. It was noted that patients with complex depression showed engagement 
difficulties; they struggled to develop therapeutic relationship and consider alternative coping strategies. Insights into unhelpful patterns of relating did not seem enough to bring interpersonal change over 16 sessions. Whilst the patients typically found the identification of the IPAF (Lemma et al, 2011a) helpful, more time was needed for the working with the maladaptive relational patterns.

Furthermore, added benefits of utilising mentalization strategies in the original DIT model were noted. It was also observed that more time was required to foster therapeutic alliance and working with defences and relational anxieties before the work on ending and separation could begin. The need for developing an augmentation of the DIT model was recognised to provide a set of guidance for its application with complex depression, which could then be subjected to research.

Following discussion of these clinical observations with the model originators, a pilot was set up for the adaptation of the 16-session DIT model to test its effectiveness. This was done in three stages: (a) adaptation of the DIT model and development of the DITCC manual by the originators; (b) pre-pilot and initial testing out stage (16 weekly sessions followed by 8 fortnightly and 2 monthly follow-ups; (c) pilot stage with further adaptations in response to the initial findings (20 weekly sessions followed by 6 fortnightly sessions and 2 monthly followups). The pilot was set up as a part of routine clinical practice; hence, it did not have the same rigour as of a research trial.

The section below provides an overview of the DITCC model, description of the pilot and its preliminary findings.

\section{DITTCC}

DITCC, like the original DIT model for mood disorders (Lemma et al., 2011a), rests on the assumption that the patient's interpersonal context is central to understanding his behaviour and experience. Current relationships can constrain an individual's capacity to function adequately. Experiencing their capacity as limited, patients feel unable to alter their personal situations, feeling ultimately trapped, helpless and denuded of resources.

The mistrust that an individual feels towards their social context can cause relative petrification; individuals are no longer able to respond in an open and flexible way to their (changing) social environment due to epistemic mistrust (Fonagy \& Allison, 2014). Rather they impose a set of tried and tested expectations, well-honed in other relationship contexts, but not responsive to any new social information that could generate change in those expectations. Particular patterns of social interaction are created that persistently devalue the relationships. Reactions may be provoked in others that generate criticism, disappointment, 
rejection, betrayal, rebuff and dismissal. These in turn create a loss of faith and trust, which precludes meaningful social experience that could be used for self-correction. The experience of social isolation is a common consequence. In DITCC, the above-described pattern of behaviours is described as Relationship Interfering Behaviours (RIBs).

Clinical observations suggest that this state of affairs is not uncommon in complex depression that appear to be relatively inaccessible to brief therapy and is frequently seen in secondary mental health settings. Naturally, the presentation is heterogeneous with limitless diagnostic profiles, but there are key trans-diagnostic consistencies rooted in the primacy they give to maladaptive interpersonal affective mental representations retained from early relationships.

Whereas DIT assumes that bringing the patient's awareness to the maladaptive qualities of such entrenched relational patterns is sufficient to modify their expectations, DITCC assumes that additionally the individual's general attitude to learning from experience must also change. Thus, DITCC has two components to its therapeutic approach, one that overlaps with DIT principles primarily concerned with identifying an IPAF and addressing its maladaptive features. The second component directly addresses the mistrust the individual feels in relation to social communications they receive and contribute further to the RIBs.

The complex cases considered in DITCC are less amenable to the therapeutic approach described in DIT often because the therapist herself or himself, along with others in the patient's social world, is regarded with suspicion. The therapeutic relationship becomes one, which elicits mistrust. Often enough the therapist finds himself or herself part of the patient's dysfunctional social system. DITCC addresses this challenge directly by specifically focusing on enhancing the trust, which an individual has for the therapist. DITCC systematically focuses the therapist's attention, particularly in the early phases of the treatment, on enhancing the perceived value of the clinician as a source of social information for the patient. Contingent responsiveness is used so that the patient's experiences of himself or herself is accurately reflected in the relationship with the therapist. The therapist aims to create an experience of agency in the patients by enabling them to perceive with clarity the relationship between their internal states and their behaviour and broader experience in relation to this interpersonal process of serial invalidation. This intervention necessarily entails coherent understanding of this aspect of their social world and the specific thoughts, feelings, beliefs, wishes and desires which motivate it. The process is less one of challenging or demanding change than one of clarification and a general enhancing of a sense of agentiveness and control. This calls for slight modifications of the therapeutic approach by the therapist, with more emphasis on the use of mentalizing techniques; including mentalizing the transference and working with enhancing affect regulation. As in the original DIT model the use of transference interpretation is employed but with a particular attention to patient capacity to mentalize. 


\section{Difficulties with mentalizing in depression}

DITCC considers the treatment resistant features of the complex case of depression to be rooted in difficulties with mentalizing and epistemic mistrust. Mentalizing is a form of imaginative mental activity about others or oneself, namely, perceiving and interpreting human behaviour in terms of intentional mental states (e.g. needs, desires, feelings, beliefs, goals, purposes, and reasons) (Bateman \& Fonagy, 2006). Secure attachment mediates the acquisition of mentalizing and formation of epistemic trust - i.e., capacity to consider new knowledge from another person as "trustworthy, generalizable, and relevant to the self" (Fonagy \& Allison, 2014, p. 375). There are well-known features of depression that suggest substantial difficulties with mentalizing, which in turn can generate complexity, described in general terms above. It is not the scope of this paper to elaborate on the contributory factors to these difficulties. However, it is important to state that disrupted attachments and traumatic experiences, which very often are present in complex depression play an important role in compromising capacity to regulate emotions and mentalize.

The clinical consequence of mistrust and non-mentalizing is not simply a lack of therapeutic response, it is also an increased risk of suicide (Bateman, O'Connell, Lorenzini, Gardner, \& Fonagy, 2016; Fonagy \& Luyten, 2009; Fonagy \& Luyten ,2018).). It is the abnormal experience of internal states, particularly psychic equivalence (concrete thinking), the teleological mode (need for action) and also the dissociation linked to pretend mode (pseudomentalizing, inconsequential talk) that increases suicidal ideation and the risk of suicide attempts. Suicidality is not restricted to this sub-group of patients. But whereas in more treatment accessible depressed patients, suicidality is directly connected to specific interpersonal circumstances, in this group of poorly mentalizing complex patients it is more often directly connected to the subjective experience itself. It is rooted in the desire to silence intense feelings of inner pain.

The most critical indicator, which represents the obstacle to therapeutic improvement, is the consequence of mistrust generated as a by-product of poor mentalizing. DITCC addresses this by attending to the patient's loss of mentalizing marked by concrete thinking, need for action and inconsequential talk (pseudo-mentalizing) and taking this as a signal of mentalization breakdown, alerting the therapist to abandon their interpretative stance and refocus their attention on helping the patient to recover their capacity to mentalize. DITCC expands the use of mentalization techniques initially in mentalizing affect and validation of interpersonal anxiety to foster understanding, trust and social learning. Once the patient is engaged with therapy, mentalization techniques are used to support this process further by reflecting on the unfolding IPAF across relationships and settings. Specifically, this is achieved 
by (a) enhancing patient's capacity to understand therapist's communications; (b) taking up work in relation to RIBs to develop capacity to listen to what the therapist is presenting and transfer this learning to other social situations. This is done with a view that patient's capacity to mentalize is generalised to other situations outside and paves way to improved communication and relationships.

\section{Who is DITCC for?}

The therapist's aim in DITCC is twofold: (a) to understand how the patient's persistent experience of social world interferes with their capacity to engage in a meaningful dialogue with the therapist that could lead to a behavioural and experiential change around the IPAF; and (b) to actively support the patient in attempting new ways of addressing the interpersonal problems linked to the onset and/or maintenance of their mood disorder through an emphasis on RIBs.

It is not the severity of depression that is critical, although severity is likely to be relatively high. Rather, DITCC is aimed at individuals with whom we anticipate significant resistance at the level of implementing interpersonal change despite the insight gained about recurrent relational patterns. It is assumed that their relative inflexibility generates RIBs that maintain the problematic relational constellation. The persistence of RIBs is seen as specifically defensive, or as a by-product of a more generalised defensive stance reflecting epistemic mistrust, a catastrophic loss of confidence in what one may learn from social experience or how one may benefit from social situations. In either case, what results is a state of affairs that precludes the translation of insight into change. RIBs are the focus of intervention in DITCC via the two simultaneously active processes of: (a) creating an increased sense of agentiveness and, through this, increasing trust in social (the therapeutic) dialogue which (b) provides insight into interpersonal processes that interfere with the possibility of change.

\section{Structure of the therapy}

DITCC comprises 26 sessions: 20 weekly sessions followed by 6 fortnightly sessions and 2 monthly follow-ups. Phase 1, "the set-up and engagement phase": 6 weekly sessions primarily concerned with establishing a working alliance and enhancing the 'therapy readiness' of the patient. Phase 2, "the insight phase": 14 weekly sessions. Initial sessions in this phase are devoted to the development of the IPAF and setting goals (maximum 3 sessions), while the remaining 11 middle phase sessions are focused on working through the IPAF and paying close attention to the RIBs. Phase 3 "the work-through and ending phase": 6 bi-weekly sessions that focus actively on helping the patient implement changes. It pays attention to the affective experience of the greater challenges of translating the generated insights into 
interpersonal changes and of managing the eventual ending. Follow-up sessions are aimed at supporting wellbeing and transition.

\section{Pilot Study}

The pilot was run in secondary care psychological services in London Borough of Newham, which offers a range of individual, family and group therapeutic interventions to people with complex, long-term and enduring mental health problems. Given the cultural diversity and socio-economic challenges in Newham, attention to culturally sensitive whole person approach was carefully considered in designing of the pilot.

The main author who led and supervised the DITCC pilot was joined by four clinicians: two psychodynamic psychotherapists, one counselling psychologist and one clinical psychologist ${ }^{1}$. The pilot lead and one of the psychotherapists were DIT trainers and supervisors, and the other three clinicians were trained in DIT prior to the start of the pilot. The pilot started in September 2016 ended in June $2018^{2}$.The project team met weekly to address emerging operational and clinical matters including setting up suitability criteria, recruitment and evaluation strategy.

\section{Assessing suitability for DITCC}

A guide for suitability for DITCC which privileged formulation-based approach over the psychiatric diagnostic categories was developed. The key criteria included: (a) complex depression, ${ }^{3}$ when it was anticipated that a focus on interpersonal targets would bring a positive change in presenting problems; (b) an interest in taking up this focus in their therapy work. The guide incorporated the $2^{\text {nd }}$ National Audit (Williams et al., 2016) findings, evidencing that the best indicator for psychotherapy success was a match between the offered treatment and patient's preference for therapy amongst available options. Therefore, an important part of assessing was supporting patients to make an informed choice about choosing DITCC from a range of therapies available. The following positive indicators were considered:

- Patient wanted to work towards self-understanding rather than with symptoms reduction.

- Patient recognised that their patterns of relationships were causing them distress.

\footnotetext{
${ }^{1}$ All clinicians taking part in the pilot were experienced therapist with a number of years of post qualification experience except one of the clinical psychologist who joined the team soon after qualifying.

${ }^{2}$ The pilot study had ended, since the submission of the paper.

${ }^{3}$ Complex depression defined as chronic, severe, co-morbid and disabling
} 
- They were interested in (a) exploring their early relationships (b) making links between their childhood experiences and their distress (c) beginning to change their relationships patterns.

Contra-indicators for DITCC were extensively debated. Given the comorbidity seen in referrals to the service, it seemed important that the exclusion criteria had clear links with the model theoretical underpinnings. The following contra-indications were set out:

- Florid and acute psychotic symptoms.

- Acute risk requiring crises and stabilisation focused interventions.

- Substance misuse as a primary problem requiring specialist help.

- Severe eating disorder.

- Severe end of Personality Disorders Spectrum and Severe Post-Traumatic Stress Disorder.

\section{Recruitment}

The suitability guide for DITCC was circulated among the service staff. The core team members offered consultations to the referring clinicians and potential pilot participants. Once the pilot became well embedded, the referral screening process generated increased appropriate referrals. The aim of the consultation was to assess for treatment suitability, facilitation of informed choice for therapy process and discuss the use of outcome measures for the pilot evaluation.

\section{Participants}

Over the period of September 2015 to September 2017, 40 patients were identified as potential pilot participants, 30 were offered specialist consultation and 21 were offered DITCC (Figure 1). Four patients were still undergoing treatment at the time of writing this paper but were included in the session-by-session analysis. Pre- and post-treatment data from the 15 treatment completers ${ }^{4}$ was used in the group-level and individual-level change analyses. Data for the 15 completers and 4 patients still undergoing therapy was used in the inter-session change analyses. Those undergoing treatment had data for a minimum of 16 sessions and up to 20 sessions. A sensitivity analysis excluding non-completers from the inter-session analyses was run.

\footnotetext{
${ }^{4}$ One patient did not have CORE-34 post-treatment data.
} 


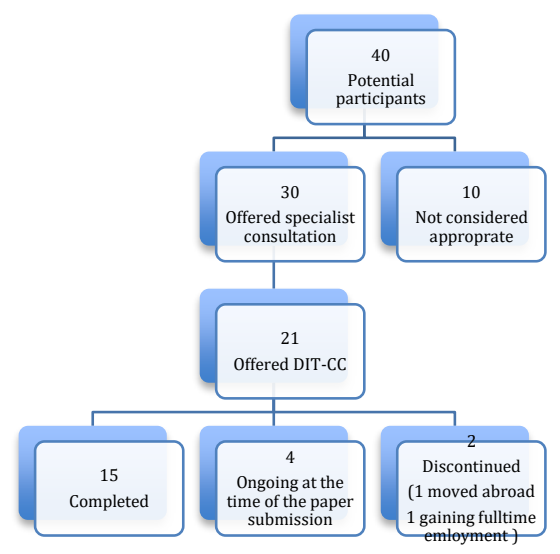

Demographic data was gathered from the service electronic information system and patients' reports during assessment and therapy (Table 1).

Table 1: Demographic Data

\begin{tabular}{|c|c|}
\hline Gender & Female $71 \%$ \\
Male $29 \%$
\end{tabular}

Further information describing patients' past and present significant life events and comorbid diagnoses are provided in Table 2. 
Table 2: Patients' Characteristics

\begin{tabular}{|c|c|}
\hline Patients' Characteristics (n, 215) & $\%$ \\
\hline Emotional deprivation and abuse from a significant carer age 0-7 & $81 \%$ \\
\hline $\begin{array}{c}\text { Traumatic experiences outside caring relationships (e.g., chronic } \\
\text { pain condition, frequent displacement age 0-7) }\end{array}$ & $43 \%$ \\
\hline $\begin{array}{c}\text { Adult experiences of unstable relationships, emotional and } \\
\text { physical abuse }\end{array}$ & $100 \%$ \\
\hline $\begin{array}{c}\text { Emotionally Unstable Personality Disorder } \\
\text { High emotional deregulation, relationship instability, BPD traits } \\
\text { (not formally assessed for PD diagnosis) }\end{array}$ & $29 \%$ \\
\hline Comorbid Generalised Anxiety & $100 \%$ \\
\hline Chronic Depression & $100 \%$ \\
\hline Emotional and relationships problems for over 10 years & $90 \%$ \\
\hline Accessing mental health services since childhood & $33 \%$ \\
\hline Self-harm & $33 \%$ \\
\hline Past suicidal crises & $38 \%$ \\
\hline Forensic history & $5 \%$ \\
\hline Past substance misuse & $52 \%$ \\
\hline Reliance on substance misuse at the start of treatment & $38 \%$ \\
\hline Past engagement with mental health services & $100 \%$ \\
\hline Prescribed antidepressants at the beginning of the treatment & $85 \%$ \\
\hline Past significant engagement with talking therapies & $33 \%$ \\
\hline
\end{tabular}

As for reports of physical health problems and disability: $38 \%$ of patients reported chronic physical health problems (Chronic Obstructive Pulmonary Disease $\mathrm{N}=1$, fibromyalgia: $\mathrm{N}=2$, endometriosis, $\mathrm{N}=1$; chronic fatigue syndrome, $\mathrm{N}=1$; chronic back pain, $\mathrm{N}=1$; spina bifida, $\mathrm{N}=1$; rheumatism $\mathrm{N}=1$ ). One patient reported impaired cognitive function and 2 patients had been given a diagnosis of Autism Spectrum Disorder.

Strategy for evaluation of the pilot

The pilot aim was to develop DITCC and test out its effectiveness . Careful consideration was given to designing the evaluation strategy including: balancing the need for the use of measures allowing research comparability and; the need for the use of sensitive measures to the changes facilitated by the piloted treatment. The development of the strategy for evaluation was an iterative process which involved on-going reviews of the observed processes facilitated in DITCC and their symptomatic, functional and interpersonal manifestations.

The Patient Health Questionnaire (PHQ-9; Kroenke, Spitzer, \& Williams, 2001) and the Generalised Anxiety Disorder (GAD-7; Spitzer, Kroenke, Williams, \& Löwe, 2006) were used to measure symptomatic change at each session. The PHQ-9 is a 9-item screening tool for depression. Scores range from $0-27$, with scores of 5, 10, 15, and 20 representing clinical cut-

\footnotetext{
${ }^{5}$ Number offered DITCC
} 
offs for mild, moderate, moderately severe, and severe depression, respectively. The GAD-7 is a 7 -item screening tool for generalized anxiety. Scores range from 0 to 21 , with scores of 5 , 10 , and 15 representing clinical cut-offs for mild, moderate, and severe anxiety, respectively. Both the PHQ-9 and GAD-7 show sensitivity and specificity rates $>80 \%$, good internal consistency (Cronbach's $\alpha>$.88), and are sensitive to therapeutic change (Kroenke, Spitzer, Williams, \& Löwe, 2010). The PHQ-9 and GAD-7 were used to increase the comparability of our results with the existent body of evidence for treatments of depression offered in the UK.

The authors shared a concern that while the PHQ-9 and GAD-7 may be sensitive to symptomatic change, they may not capture changes in broader functioning targeted by DITCC, such as global well-being and interpersonal functioning. Therefore, two broader measures of functioning at pre- and post-treatment: the CORE-34 ${ }^{6}$ (CORE-34; Evans et al., 2000; Barkham, Mellor-Clark, Connell, \& Cahill, 2006) and the Schwartz Outcome Scale-10 ${ }^{7}$ (SOS-10; Blais et al., 1999) were included. The CORE-34 is a 34-item self-report measure of global distress, including subjective well-being, commonly experienced problems or symptoms, social/life functioning and risk to self and others. Total scores are divided by the number of items and multiplied by 10 , so that scores range from $0-40$. Scores of $10,15,20$, and 25 reflect clinical cut-offs for mild, moderate, moderately severe, and severe levels of distress, respectively. The measure is suitable for use as an initial screening tool and for assessing response to psychological therapy across a wide range of service types (Barkham et al., 2006). The CORE-34 is sensitive to change and has high internal and test-retest reliability (Evans et al., 2000). The SOS-10 is a measure of psychological well-being, including life satisfaction, interpersonal effectiveness, positive self-appraisal, optimism, and the absence of psychiatric symptoms (Blais et al., 1999). Scores range from $0-60$, with scores $<41$ falling within the clinical range. The SOS-10 shows good construct validity (Haggerty et al., 2012) and reliability (Young, Waehler, Laux, McDaniel, \& Hilsenroth, 2003).

\section{Data Analysis}

Treatment effects were assessed at three levels: the group level, individual level, and intersession level.

Group-level treatment effects were assessed with paired-samples $t$ tests of pre-post mean differences for treatment completers $(N=15)$ on the CORE-OM, PHQ-9, GAD-7 and SOS10. Effect sizes were calculated using Hedges $g$, which is preferable to Cohen's $d$ in small samples (Hedges, 1981). 
Individual-level treatment effects were calculated using Jacobson and Truax's (1991) criteria for reliable and clinically significant change for treatment completers $(N=15)$. Clinically significant improvement was defined as pre-treatment and post-treatment scores above and below 10 on the CORE-34 (Barkham, et al., 2006), 41 on the SOS-10 (Blais et al., 2013; Haggerty et al., 2012), 10 on the PHQ-9 and GAD-7 A reliable change index for the COREOM, SOS-10, PHQ-9, and GAD-7 was calculated using the current sample's pre-treatment standard deviation on each measure, and reliability estimates (Cronbach's $\alpha$ ) from each measure's initial validation study (e.g., $\alpha_{\mathrm{CORE}-\mathrm{OM}}=.94$, Evans et al., 2002; $\alpha_{\mathrm{sos}-10}=.93$, Blais et al., 1999; $\alpha_{\mathrm{PHQ}-9}=.89$, Kroenke et al., 2001; $\alpha_{\mathrm{GAD}-7}=.89$, Löwe et al., 2008).

Reliable improvement (or deterioration) was defined as pre-to-post difference scores $>5.11$ (or <-5.11) on the CORE-OM, > 5.17 (or <-5.17) on the PHQ-9, > 4.02 (or <-4.02) on the GAD-7, and <-8.22 (or > 8.22) on the SOS-10 (clinical gain is indicated by an increase in scores). No reliable change was defined as pre-post difference scores between -5.11-5.11 on the CORE-OM, -5.17-5.17 on the PHQ-9, -4.02-4.02 on the GAD-7, and -8.22-8.22 on the SOS-10.

Inter-session treatment effects were estimated using linear mixed-effects growth curve models using all available data for both treatment completers and non-completers $(N=19)$. Data were arranged in long-format, where each row held a score for each participant at each time-point. Models included the fixed effects of time (Baseline was coded as 0 . Weeks 1-20 were coded as 1-20. Weeks 21-26 were coded as 22-32 in increments of 2 to reflect the biweekly interval), time-squared (e.g., $t 0=0, t 1=1, t 2=4, \ldots t 32=1024$ ), age (centred), sex, educational attainment, employment status, therapist ID, the number of sessions offered (centred; $M=25$, $S D=6$, range $=11-26)$, and the number of sessions missed $(M=2, S D=3$, range $=0-10)$. Baseline scores and linear time effects varied across patients using random effects, which were free to co-vary. A random slope for quadratic time was not included as it did not improve the fit of the model predicting PHQ-9 scores $\left(X^{2}(1)=.60=p>.05\right)$ or GAD-7 scores $\left(X^{2}(1)=\right.$ $1.35=p>.05)$.

\section{Results}

\section{Group-Level Change}

Mean scores for treatment completers and paired sample $t$-tests are shown in Table 3. Large and significant reductions were found on the PHQ-9, GAD-7, and CORE-34, and a large and significant increase was found on the SOS-10. Post-hoc power analyses indicated that we were highly powered to detect pre-post differences on all measures $(\beta=.93-.99)$.. 
Table 3. Means and standard deviations (in brackets) for each outcome measure at preand post-treatment for treatment completers $(N=15)$.

\begin{tabular}{lcccccc}
\hline \multirow{2}{*}{ Measure } & \multicolumn{2}{c}{ Treatment Phase } & \multicolumn{3}{c}{} \\
\cline { 2 - 7 } & Pre & Post & $t$ & $p$ & $95 \% \mathrm{Cl}$ & $g[95 \% \mathrm{Cl}]$ \\
\hline PHQ-9 & $16.66(5.62)$ & $10.53(1.43)$ & 3.23 & .006 & {$[2.07,10.18]$} & $1.04[0.21,1.86]$ \\
GAD-7 & $15.28(4.37)$ & $8.08(5.54)$ & 3.37 & .005 & {$[2.63,11.82]$} & $1.37[0.20,2.55]$ \\
CORE-34 & $23.05(7.53)$ & $14.82(7.98)$ & 3.97 & .002 & {$[3.75,12.71]$} & $1.00[0.36,1.64]$ \\
SOS-10 & $16.46(7.41)$ & $30.47(12.72)$ & -5.00 & $<.001$ & {$[-20.00,-8.00]$} & $-1.19[-1.88,-0.54]$ \\
\hline \hline
\end{tabular}

Note. PHQ-9 = Patient Health Questionnaire-9; GAD-7 = Generalized Anxiety Disorder-7;

CORE-34 = CORE Outcome Measure-34; SOS-10 = Schwartz Outcome Scale-10.

\section{Individual-level Change}

Rates of clinically significant improvement and reliable changeare reported in Table 4.

More than half of patients on the GAD-7, and around one-third on the PHQ-9, CORE-34, and SOS-10, were no longer in the clinical range. Roughly two-thirds of patients showed reliable improvement across measures. Only two patients showed reliable deterioration on the GAD7, and one on the PHQ-9. And around one-third of patients did not show reliable change on any of the measures.

Table 4: The number and percentage of treatment completers showing reliable and clinically significant change.

\begin{tabular}{lcccc}
\hline \multicolumn{1}{c}{ Measure } & CSC & Improvement & Deterioration & No Change \\
\hline PHQ-9 $(N=15)$ & $5(33 \%)$ & $10(67 \%)$ & $1(7 \%)$ & $4(27 \%)$ \\
GAD-7 $(N=15)$ & $9(60 \%)$ & $11(73 \%)$ & $2(13 \%)$ & $2(13 \%)$ \\
CORE-34 $(N=14)$ & $4(29 \%)$ & $10(71 \%)$ & $0(0 \%)$ & $4(29 \%)$ \\
SOS-10 $(N=15)$ & $4(27 \%)$ & $9(60 \%)$ & $0(0 \%)$ & $6(40 \%)$ \\
\hline \hline
\end{tabular}

Note. CSC = clinically significant change; PHQ-9 = Patient Health Questionnaire-9; GAD-7 = Generalized Anxiety Disorder-7; CORE-34 = CORE Outcome Measure-34; SOS-10 = Schwartz Outcome Scale-10.

\section{Inter-session Change}

Table 6 shows the observed means and standard deviations for the PHQ-9 and GAD-7 at each phase of therapy using all available data . Linear mixed-effects growth curve models estimating session-by-session change showed a significant linear decline in PHQ-9 scores over the study period ( $B=-.37, p<.001,95 \% \mathrm{Cl}[-.54,-.20]$ ). In other words, PHQ-9 scores were predicted to steadily decrease by .37 points each session (see Figure 2 ). The quadratic 
effect of time-squared was small but significant $(B=.01, p<.05,95 \% \mathrm{Cl}[.001, .01])$; that is, the rate at which PHQ-9 scores declined slowed down over time. There were small differences in the rates of decline between patients $\left(v_{1 j}=.03,95 \% \mathrm{Cl}[.01, .07]\right)$, but they differed substantially in their baseline values $\left(u_{1 j}=8.00,95 \% \mathrm{Cl}[2.40,26.65]\right)$.

Patients also showed a significant linear decline in GAD-7 scores $(B=-.49, p<.001,95 \% \mathrm{Cl}$ $[-.65,-.33])$, as well as a small but significant quadratic increase decline over time $(B=.01, p$ $<.05,95 \% \mathrm{Cl}[.005, .02])$. That is, GAD-7 scores decreased almost half a point with each session early on, but the rate of decline slowed down at later sessions (see Figure 3). Like the PHQ-9, variation in the rates of decline was minimal $\left(v_{1 j}=.03,95 \% \mathrm{Cl}[.01, .07]\right)$, but patients differed in their initial severity $\left(u_{1 j}=15.17,95 \% \mathrm{Cl}[6.01,38.28]\right)$.

Results were maintained when participants who were still undergoing treatment $(n=5)$ were excluded. PHQ-9 scores continued to decline over time $(B=-.31, p<.001,95 \% \mathrm{Cl}[-.49$, $.14])$ and the rate of change continued to decrease $(B=.01, p<.05,95 \% \mathrm{Cl}[.001, .01])$. Moreover, GAD-7 scores continued to decline over time $(B=-.51, p<.001,95 \% \mathrm{Cl}[-.69$, $.35])$ at a slowing rate $(B=.01, p<.001,95 \% \mathrm{Cl}[.01, .02])$.

Table 5. Means and standard deviations for the PHQ-9 and GAD-7 at each phase of DITCC using all available cases $(N=19)$.

\begin{tabular}{|c|c|c|c|c|c|c|c|c|}
\hline & \multicolumn{8}{|c|}{ Phase (Session) } \\
\hline & \multicolumn{2}{|c|}{ Baseline } & \multicolumn{2}{|c|}{$\begin{array}{c}\text { Set-up \& } \\
\text { Engagement (1-6) }\end{array}$} & \multicolumn{2}{|c|}{$\begin{array}{c}\text { Insight } \\
(7-20)\end{array}$} & \multicolumn{2}{|c|}{$\begin{array}{l}\text { Work-through \& } \\
\text { Ending }(21-26)\end{array}$} \\
\hline & $M$ & $S D$ & $M$ & $S D$ & $M$ & $S D$ & $M$ & $S D$ \\
\hline PHQ-9 & 16.66 & 5.62 & 15.74 & 6.83 & 13.59 & 6.62 & 12.23 & 6.53 \\
\hline GAD-7 & 15.28 & 4.37 & 13.72 & 5.62 & 10.92 & 5.564 & 10.00 & 5.72 \\
\hline
\end{tabular}

Figure 2. Predicted and observed mean PHQ-9 scores at each weekly session (timepoints 1-20) and bi-weekly session (time-points 21-26) using all available data ( $N=19$ ). Errors bars reflect standard deviations and dotted lines reflect standard errors of the mean. 


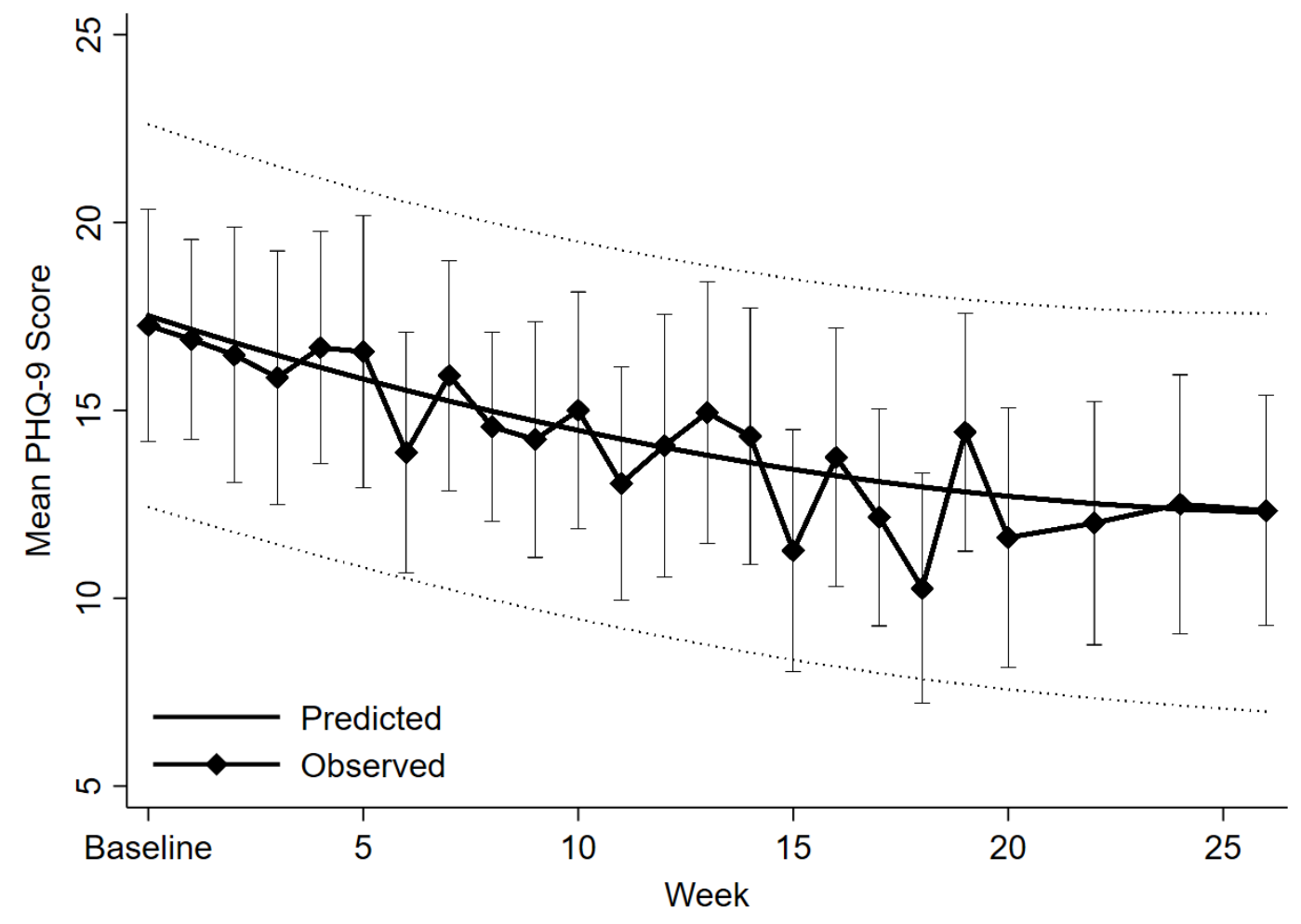

Figure 3. Predicted and observed mean GAD-7 scores at each weekly session (timepoints 1-20) and bi-weekly session (time-points 21-26) using all available data $(N=19)$. Errors bars reflect standard deviations and dotted lines reflect standard errors of the mean.

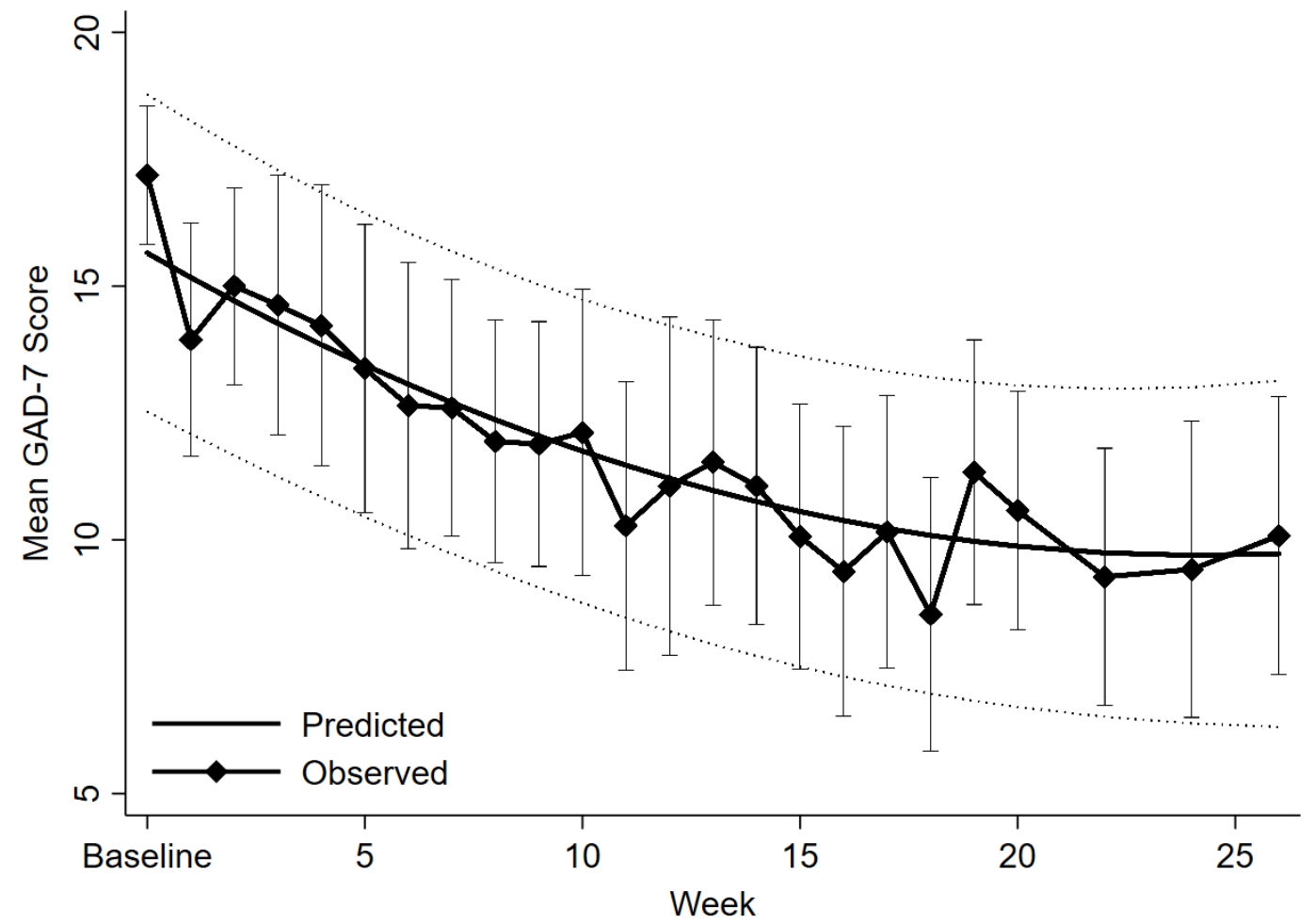




\section{Discussion}

DIT formulates the presenting symptoms of depression as responses to interpersonal difficulties and perceived threats to attachments and hence also as threats to the self (Lemma et al, 2011 a). DITCC additionally focuses on change in individual's general attitude to learning from experience. The model pays a particular attention to epistemic mistrust, which often leads to experience of social isolation in complex cases of depression. This is done by focusing on: (a) enhancing capacity to mentalize and creating a mentalized version of the dysfunctional communication patterns with a focus on therapeutic relationship; (b) creating an increased sense of agentiveness and, through this, increasing trust in social (therapeutic) dialogue which; (c) providing insight into interpersonal processes that interfere with the possibility of change.

The aim of the pilot was to consider the DITCC effectiveness with patients presenting with complex depression. Session by session outcome monitoring was used to examine the process of change across various stages of therapy. The initial analysis of the data included in this paper, provides preliminary evidence for the effectiveness of DITCC for complex depression.

The project found that measures of depression, anxiety, and overall clinical severity declined from pre-to-post therapy across the sample. Furthermore, psychological well-being showed a large pre-post treatment increase. While these group-level effects demonstrate the effectiveness of DITCC in treating multiple areas of psychological functioning, they are not sensitive to individual changes. In an effort to triangulate assessment of treatment effects, reliable and clinically significant change were calculated. These analyses revealed more modest, but no less promising, effects of DITCC. For instance, at least half of patients showed reliable improvement in depression, anxiety, general clinical severity, and psychological wellbeing. Improvement in depression was deemed clinically significant for up to half of patients (i.e. patients reached a non-clinical level), which is a respectable outcome given their initial severity. Furthermore, very few (1\%) showed reliable deterioration. There was, however, an appreciable minority of patients who showed no reliable change, particularly in general clinical severity $(44 \%)$. These rates are similar to past non-controlled trials for treatment-resistant depression (Brakemeier et al., 2015), and indicate general barriers to treatment. Lastly, session-by-session measures of depression and anxiety confirm the changes observed at the group and individual levels. That is, depression and anxiety declined rather steadily and consistently across patients over study period. Decline did slow down over the treatment period, but this is typical of treatment studies, particularly when the termination of therapy is approaching. It will be important to determine the extent to which these promising findings are 
due to the natural passage of time or generally being helped through a randomized controlled trial with an active control group.

Despite the obvious limitations of the study, DITTCC appeared to have face validity across patients from diverse socioeconomic and ethnic backgrounds. Initial reports from the patients have been encouraging. Therapist's experience had been positive indicating that therapists from different background can be effectively trained in DITTCC with a provision of regular supervision to help with the dynamic focus, model adherence and management of high risk patients. Results need to be interpreted with caution due to the obvious limitation arising from the small sample size, limited analysis and the lack of follow-up data. The pilot was set up as an observational small-scale study. Its methodology and findings are unlikely to have similar level of rigour as expected in research trials. There are many questions which require further exploration such as differential effects across patients; what factors supported and hindered change and engagement; what part the focus on choice of therapy played in treatment outcomes; what aspects of DITCC were experienced as more or less helpful across the three phases by patients; what level of change will be sustained over the course of time. Further research would shed some further light on some of these questions.

\section{Implications of the pilot}

The pilot has demonstrated some initial trends regarding DITCC potential effectiveness. Future work on the pilot will include: (a) fuller analysis of the quantitative data (b) analyses of the qualitative data (patients' interviews and the detailed case material). The results of those analyses will be used to generate questions for further research and to work towards further refinement of the model.

The pilot was set up was tested in a service which like many other health services needed to respond to pressures around mismatch between demand and resources and meet quality assurance standards. The pilot was conducted without additional resources and had demonstrated that advancement in clinical practice and model development is possible as part of routine practice with a clear set of protocols and collaboration with academic experts. The pilot had shown that session-by-session measures could be administered with complex diagnostic presentations and their dynamic use in therapy could be both beneficial for patients and therapists.

Results of the pilot need to be interpreted with caution, given its methodological limitations, including a small sample size, limited analysis, the lack of follow-up data, uncontrolled design, lack of comparison group and model adherence ratings. The pilot's initial findings provide a template for further testing out of the DITCC model. Implications for training in DITCC would need to be looked into to consider options based on the DIT competency-based approach. 


\section{References:}

1. Abbass, A. A., Hancock, J. T., Henderson, J., \& Kisely, S. (2006). Short-term psychodynamic psychotherapies for common mental disorders. Cochrane Database of Systematic Reviews, Issue 4, Article No. CD004687.

2. Andrews, G. (2008). Reducing the burden of depression. Canadian Journal Psychiatry, 53 (7), 420-427.

3. Barkham, M., Mellor-Clark, J., Connell, J., \& Cahill, J. (2006). A core approach to practicebased evidence: A brief history of the origins and applications of the CORE-OM and CORE System. Counselling and Psychotherapy Research, 6 (1), 3-15.

4. Barkham, M., \& Saxon, D. (2018). The effectiveness of high-intensity CBT and counselling alone and following low-intensity CBT: a re-analysis of the 2nd UK National Audit of Psychological Therapies data. BMC Psychiatry, 18 (321),1-5.

5. Bateman, A., \& Fonagy, P. (2006). Mentalization based treatment: a practical guide. Oxford: Oxford University Press.

6. Bateman, A., O'Connell, J., Lorenzini, N., Gardner, T., \& Fonagy, P. A. (2016). Randomized controlled trial of mentalization-based treatment versus structured clinical management for patients with comorbid borderline personality disorder and antisocial personality disorder. BMC Psychiatry, 16, 1-11.

7. Blais, M. A., Lenderking, W. R., Baer, L., deLorell, A., Peets, K., Leahy, L., \& Burns, C. (1999). Development and initial validation of a brief mental health outcome measure. Journal of Personality Assessment, 73(3), 359-373.

8. Blais, M. A., Malone, J. C., Stein, M. B., Slavin-Mulford, J., O'Keefe, S. M., Renna, M., \& Sinclair, S. J. (2013). Treatment as usual (TAU) for depression: A comparison of psychotherapy, pharmacotherapy, and combined treatment at a large academic medical center. Psychotherapy, 50(1), 110.

9. Bower, P., Gilbody, S., Richards, D., Fletcher, J., \& Sutton, A. (2006). Collaborative care for depression in primary care. Making sense of a complex intervention: systematic review and meta-regression. British Journal of Psychiatry, 189, 484-493.

10. Brakemeier, E. L., Radtke, M., Engel, V., Zimmermann, J., Tuschen-Caffier, B., Hautzinger, M., \& Normann, C. (2015). Overcoming treatment resistance in chronic depression: a pilot study on outcome and feasibility of the cognitive behavioral analysis system of psychotherapy as an inpatient treatment program. Psychotherapy and Psychosomatics, 84(1), 51-56.

11. Buckman, J.E.J., Underwood, A., Clarke, K., Saunders, R., Hollon, S. D., Fearon, P. \& Pilling, S. (2018). Identifying risk factors for relapse and recurrence of depression in adults and considering how they operate: a four-phase systematic review and meta-synthesis. Clinical Psychology Review, 64, 13-38. 
12. Buszewicz, M., Griffin, M., McMahon, E. M., Beecham, J., \& King, M. (2010). Evaluation of a system of structured, pro-active care for chronic depression in primary care: a randomised controlled trial. BMC Psychiatry, 10, 61.

13. Chen, C. K., \& Dognin, J. S. (2017). Addressing the influence of hegemonic masculinity on veterans through brief dynamic interpersonal therapy. Psychology of Men \& Masculinity, 18(3), 238-242.

14. Chen, C. K., Ingenito, C. P., Kehn, M. M., Nehrig, N., \& Abraham, K. S. (2017). Implementing brief Dynamic Interpersonal Therapy (DIT) in a VA Medical Center. Journal of Mental Health, 7(12), 1-8.

15. Delfstra, G., \& Van Rooij, W. (2015). Dynamic Interpersonal Therapy (DIT): application in the treatment of medically unexplained somatic symptoms. Psychoanalytic Psychotherapy, 29(2), 171-181.

16. Dognin, J. S., \& Chen, C. K. (2018). The secret sorrows of men: impact of Dynamic Interpersonal Therapy on 'masculine depression'. Psychoanalytic Psychotherapy, 32 (2), 181-196

17. Douglas, A., Ablett-Tate, N., \& Chadd, N. (2016). Dynamic interpersonal therapy in an NHS tertiary level specialist psychotherapy service. Psychoanalytic Psychotherapy, 30(3), 223-239.

18. Eaton, W. W., Shao, H., Nestadt, G., Lee, B. H., Bienvenu, O. J., \& Zandi, P. (2008). Population-based Study of first onset and chronicity in major depressive disorder. Archives of General Psychiatry, 65 (5), 513-520.

19. Evans, C., Mellor-Clark, J., Margison, F., Barkham, M., Audin, K., Connell, J., and McGrath, G. (2000). CORE: Clinical Outcomes in Routine Evaluation. Journal of Mental Health, 9(3), 247-255.

20. Ferrari, A. J., Somerville, A. J., Baxter, A. J., Norman, R., Patten, S.B., Vos, T., \& Whiteford, H.A. (2013). Global variation in the prevalence and incidence of major depressive disorder: a systematic review of the epidemiological literature. Psychological Medicine, 43:471-81.

21. Fonagy, P., \& Allison, E. (2014). The role of mentalizing and epistemic trust in the therapeutic relationship. Psychotherapy, 51(3), 372-380.

22. Fonagy, P., \& Luyten, P. (2009). A developmental, mentalization-based approach to the understanding and treatment of borderline personality disorder. Development and Psychopathology, 21, 1355-1381.

23. Fonagy, P., \& Luyten, P. (2018). Attachment, mentalizing, and the self. In W. J. Livesley \& R. Larstone (eds.), Handbook of Personality Disorders (pp. 123-140). New York; Guilford Press.

24. Haggerty, G., Aflatoon, K., Ghosh, K., Babalola, R., Ahmed, Z., \& Alois, A. (2012). Construct validity of the Schwartz Outcome Scale: Validation using a 28-day inpatient 
chemical rehabilitation patient sample. The Journal of Nervous and Mental Disease, 200(4), 343-348.

25. Haggerty, G., Kahoud, D., Walsh, E., Forlenza, N., Ahmed, Z., \& Ditkowsky, K. (2013). A brief measure of psychological health and well-being: initial validation of the Schwartz outcome scale for an adolescent inpatient sample. Journal of Nervous and Mental Disease, 201(3), 216-221.

26. Hedges, L. V. (1981). Distribution theory for Glass's estimator of effect size and related estimators. Journal of Educational Statistics, 6(2), 107-128.

27. Hollon, S. D., \& Ponniah, K. (2010). A review of empirically supported psychological therapies for mood disorders in adults. Depression and Anxiety, 27(10), 891-932.

28. Jacobson, N. S., \& Truax, P. (1991). Clinical significance: a statistical approach to defining meaningful change in psychotherapy research. Journal of Consulting and Clinical psychology, 59(1), 12-19.

29. Johnsen, T. J., \& Friborg, O. (2015). The Effects of Cognitive Behavioral Therapy as an Anti-Depressive Treatment is Falling: A Meta-Analysis. Psychological Bulletin. American Psychological Association,1-22.

30. Kessler, R. C., Berglund, P., Demler, O., Jin, R., Koretz, D., Merikangas, K. R., \& Wang, P. S. (2003). The epidemiology of major depressive disorder: results from the National Comorbidity Survey Replication (NCS-R). Journal of the American Medical Association, 289, 3095-3105.

31. Kroenke, K., Spitzer, R. L., \& Williams, J. B. (2001). The PHQ-9: Validity of a brief depression severity measure. Journal of General Internal Medicine, 16, 606-613.

32. Kroenke, K., Spitzer, R. L., Williams, J. B., \& Löwe, B. (2010). The patient health questionnaire somatic, anxiety, and depressive symptom scales: a systematic review. General Hospital Psychiatry, 32(4), 345-359.

33. Lépine, J-P., \& Briley, M. (2011). The increasing burden of depression. Neuropsychiatric Disease and Treatment, 7, 3- 7.

34. Lemma, A., Target, M., \& Fonagy, P. (2011a). Dynamic interpersonal therapy: A clinician's guide. Oxford: Oxford University Press.

35. Lemma, A., Target, M., \& Fonagy, P. (2011b). The development of a brief psychodynamic intervention (Dynamic Interpersonal Therapy) and its application to depression: A pilot study. Psychiatry: Interpersonal and Biological Processes, 74(1), 41-48.

36. Lemma, A., \& Fonagy, P. (2013). Feasibility study of a psychodynamic online group intervention for depression. Psychoanalytic Psychology, 30(3), 367-380.

37. Leonidaki, V., Lemma, A. \& Hobbis, I. (2016). Clients' experiences of dynamic interpersonal therapy (DIT): opportunities and challenges for brief, manualised psychodynamic therapy in the NHS. Journal of Psychoanalytic Psychotherapy, 30(1), 4261. 
38. Löwe, B., Decker, O., Müller, S., Brähler, E., Schellberg, D., Herzog, W., \& Herzberg, P. Y. (2008). Validation and Standardization of the Generalized Anxiety Disorder Screener (GAD-7) in the General Population. Medical Care, 46(3), 266-274.

39. McPherson, S., Cairns, P., Carlyle, J., Shapiro, D. A., Richardson, P., \& Taylor, D. (2005). The effectiveness of psychological treatments for treatment-resistant depression: a systematic review. Acta Psychiatrica Scandinavica, 111(5), 331-340.

40. McPherson, S, Rost, F., Town, J., \& Abbass, A., (2018) Epistemological flaws in NICE review methodology and it's impact on recommendations for psychodynamic psychotherapies for complex and persistent depression. Psychoanalytic Psychotherapy, 32(2), 102-121.

41. McPherson, S., \& Armstrong, D. (2009). Negotiating 'depression' in primary care: a qualitative study. Social Science and Medicine, 69 (8), 1137-1143.

42. Mollon, P. (2009). The NICE guidelines are misleading, unscientific, and potentially impede good psychological care and help. Psychodynamic Practice, (15)1, 9-24.

43. Moorey, S. (2010). Cognitive Behaviour Therapy and psychoanalysis. In A. Lemma \& M. Patrick (Ed.), Off the couch: contemporary psychoanalytic applications (pp. 194-211). Hove, East Sussex: Routledge.

44. Morriss, R., Marttunnen, S., Garland, A., Nixon, N., McDonald, R., Sweeney, T. Yang, M. (2010). Randomised controlled trial of the clinical and cost effectiveness of a specialist team for managing refractory unipolar depressive disorder. BMC Psychiatry, 10, 1-11.

45. Mueller, T. I., Leon, A.C., Keller, M.B., Solomon, D. A., Endicott, J, Coryell, W., ... Maser, J,D. (1999). Recurrence after recovery from major depressive disorder during 15 years of observational follow-up. American Journal of Psychiatry, 156, 1000-1006

46. National Health Service [NHS], (2019). The NHS long term plan. www.longtermplan.nhs.uk.

47. NHS Digital (2017-18). Psychological Therapies: Annual Report on the use of IAPT services, England.

48. NHS England/Health Education England. 2015 Adult IAPT Workforce Census Report. London: NHS England; 2016.

49. NHS (2014). Five Years Forward View. www.england.nhs.uk/five-year-forward-view.

50. Paykel, E. S., Scott, J., Cornwall, P. L., Abbott, R., Crane, C., Pope, M., \& Johnson, A. L. (2005). Duration of relapse prevention after cognitive therapy in residual depression: follow-up of controlled trial. Psychological Medicine, 35(1), 59-68.

51. Perfect, D., Jackson, C., Pybis, J., \& Hill, A. (2016). Choice of therapies in IAPT: An overview of the availability and client profile of step 3 therapies, British Association for Counselling \& Psychotherapy.

52. Pybis, J., Saxon, D., Hill, A., \& Barkham, M. (2017). The comparative effectiveness and efficiency of cognitive behaviour therapy and counselling in the treatment of depression: 
evidence from the $2^{\text {nd }}$ UK National Audit of Psychological Therapies. BMC Psychiatry, 17: 215,1-13.

53. Roth, A., \& Fonagy, P. (2005). What Works for Whom? Second edition. London: Guilford Press.

54. Selders, M., Visser, R., Rooij, W-V., Delfstra, G., \& Koelen, J. A. (2015) The development of a brief group intervention (Dynamic Interpersonal Therapy) for patients with medically unexplained somatic symptoms: a pilot study, Psychoanalytic Psychotherapy, 29 (2), $182-$ 198.

55. Spitzer, R. L., Kroenke, K., Williams, J. B., \& Löwe, B. (2006). A brief measure for assessing generalized anxiety disorder: the GAD-7. Archives of Internal medicine, 166(10), 1092-1097.

56. Stimpson, N., Agrawal, N., \& Lewis, G. (2002). Randomised controlled trials investigating pharmacological and psychological interventions for treatment-refractory depression: systematic review. British Journal of Psychiatry, 181(4), 284-294.

57. Taylor, D., Carlyle, J., McPherson, S., Rost, F., Thomas, R., \& Fonagy, P (2012). Tavistock Adult Depression Study (TADS): a randomised controlled trial of psychoanalytic psychotherapy for treatment-resistant/ treatment-refractory forms of depression. BMC Psychiatry, 12(60), 1-13.

58. Ustün, T. B., Ayuso-Mateos, J., Chatterji, S., Mathers, C. J., \& Murray, C. (2004). Global burden of depressive disorders in the year 2000. British Journal of Psychiatry, 184, 386392.

59. Westen, D., Novotny, C. M., \& Thompson-Brenner, H. (2004). The empirical status of empirically supported psychotherapies: Assumptions, findings, and reporting in controlled clinical trials. Psychological Bulletin, 130 (4), 631-663.

60. Williams, R., Farquharson, L., Palmer, L., Bassett, P., Clarke, J., Clark, D. M., \& Crawford, M. J. (2016). Patient preference in psychological treatment and associations with selfreported outcome: national cross-sectional survey in England and Wales. BMC Psychiatry, 16 (4), 1-8.

61. Vos, T., Barber, R. M., Bell, B., Bertozzi-Villa, A., Biryukov, S., Billiger, I., \& Murray, C. J., (2015). Global Burden of Disease Study 2013 Collaborators. Global, regional, and national incidence, prevalence, and years lived with disability for 301 acute and chronic diseases and injuries in 188 countries, 1990-2013: a systematic analysis for the Global Burden of Disease Study 2013. Lancet, 386, 743-800.

62. Young, J. L., Waehler, C. A., Laux, J. M., McDaniel, P. S., \& Hilsenroth, M. J. (2003). Four studies extending the utility of the Schwartz Outcome Scale (SOS-10). Journal of Personality Assessment, 80(2), 130-138.

63. Wright, D., \& Abrahams, A. (2015). An investigation into the effectiveness of Dynamic Interpersonal Therapy (DIT) as a treatment for depression and anxiety in IAPT, Psychoanalytic Psychotherapy, 29 (2) 160-170. 
